\title{
Effect of COVID-19 pandemic on stroke admissions and quality of stroke interventional treatment in Masovian Voivodeship
}

\author{
Aleksander Dębiec ${ }^{1}$, Marta Bilik ${ }^{2}$, Piotr Piasecki ${ }^{3}$, Adam Stępień ${ }^{1}$, Jacek Staszewski ${ }^{1}$ \\ ${ }^{1}$ Clinic of Neurology, Military Institute of Medicine, Warsaw, Poland \\ ${ }^{2}$ Department of Neurology with Stroke Unit, John Paul II Western Hospital, Grodzisk Mazowiecki, Poland \\ ${ }^{3}$ Department of Interventional Radiology, Military Institute of Medicine, Warsaw, Poland
}

\begin{abstract}
Aim of study. To assess the impact of the coronavirus disease 2019 (COVID-19) pandemic on the pathway of stroke interventional services and major quality indicators of stroke reperfusion therapies in Masovian Voivodeship.

Materials and methods. An exploratory retrospective analysis was performed at two comprehensive stroke centres to assess changes in stroke care between the early phase of the COVID-19 pandemic (weeks 10-18 of 2020) and the same period in 2019.

Results. Of the 419 included stroke patients, 186 (44.4\%) presented during the COVID-19 period. There was an increase in in-hospital delays for reperfusion therapies, and a significant decrease in the number of acute cerebrovascular accident admissions, predominantly related to a low number of transient ischaemic attack (TIA) admissions to hospital (-20.17\%). The delays were shorter in the mothership paradigm than in the drip-and-ship paradigm of acute stroke care (onset-to-groin 293 vs. 232 $\min , p=0.03$ ). No differences in stroke aetiology, large-vessel occlusion frequency, or severe stroke admissions in the COVID-19 period were observed.
\end{abstract}

Conclusions and clinical implications. COVID-19's emergence was correlated with a significant reduction in admissions to stroke departments, particularly for TIAs, and a prolonged delay in reperfusion stroke treatment, especially in the drip-and-ship paradigm. An educational campaign to raise public awareness of TIA and/or stroke symptoms and immediate reorganisation of stroke care during the COVID-19 era are necessary.

Key words: stroke, COVID-19, healthcare system, mechanical thrombectomy, stroke quality measures (Neurol Neurochir Pol 2021; 55 (2): 223-226)

\section{Introduction}

The World Stroke Organisation has identified a marked fall in stroke presentations and a widespread impact of the coronavirus 2019(COVID-19) pandemic on stroke services, with reduced evaluations and admissions for stroke during the first half of 2020 [1].

Despite the potential consequences of untreated or undiagnosed strokes, patients are less willing to seek medical care. This is related to the call for social distancing and increased use of remote teleconsultations recommended by the Polish Ministry of Health instead of face-to-face meetings [2,3]. The pandemic has required drastic changes in resource allocation, which can affect stroke care delivery. Knowledge of the gaps in the stroke interventional service pathway is important because of the great impact of delays on stroke outcome. Reports of the effect of COVID-19 on Polish health services in acute cerebrovascular accidents are lacking.

\section{Methods}

The aim of this study was to assess the impact of the COVID-19 pandemic on the stroke interventional service pathway and care quality indicators for stroke management. In this exploratory retrospective analysis, we collected data

Address for correspondence: Aleksander Dębiec, Clinic of Neurology, Military Institute of Medicine, Szaserow 128 Str., 04-141 Warsaw, Poland, e-mail: adebiec@wim.mil.pl 
on the number of consecutive hospital admissions of adults aged 18-plus admitted due to a final diagnosis of acute cerebrovascular accident (ischaemic stroke, haemorrhagic stroke, subarachnoid haemorrhage (SAH) and transient ischaemic attack [TIAs]), stroke subtypes (using the TOAST classification), reperfusion therapies and stroke quality measures (door-to-needle [DTN], door-to-computed tomography [DTCT], door-to-groin [DTG], onset-to-groin [OTG], effective recanalisation [TICI $2 \mathrm{~b}$ or 3], and 30-day mortality rate).

Data was collected from two comprehensive stroke centres (CSCs) covering majority of interventional stroke services for Masovian Voivodeship that serve as referral bases for 15 primary stroke centres in this region, which has a population of 800,000 , and included all patients hospitalised in weeks 10 to 18 of 2020 (the initial COVID-19 period) and the same period in 2019 (the pre-COVID-19 period). 1 March 2020 (week 10) was selected as the start date of the COVID-19 period, as this was the date when the first patient developed COVID-19 symptoms with serological confirmation of SARS-CoV-2 infection. On 20 March 2020, Poland was placed into lockdown by government, but social distancing and the cancellation of scheduled hospital admissions had been encouraged since the beginning of March. The number of stroke/TIA cases is presented as cases $/ 100,000$ inhabitants, and the population was assumed to remain stable from 2019 to 2020 because the change in population in recent years has been small, and no mass migration from cities was observed. Data was compared by univariate analysis using the Wilcoxon-Mann-Whitney test and 2-tailed Fisher's exact test using PQStat software. Differences with $p$ values $<0.05$ were considered statistically significant.

\section{Results}

Of the 419 included patients, 186 (44.4\%) presented in the COVID-19 period (Tab. 1). There was a significant decrease in the number of acute cerebrovascular accident admissions $(-20.17 \%)$ between the pre-COVID-19 and COVID-19 periods, which was related to the low number of TIA admissions, especially in weeks 12, 13 and 17 (Fig. 1). There were no differences in stroke aetiology, the frequency of large-vessel occlusions, severe stroke admissions, or reperfusion procedures between 2020 and 2019. However, major delays (DTCT, DTN, DTG, and OTG) in patients receiving reperfusion treatment significantly increased during the COVID-19 period. There was also a significant increase in OTG time for patients treated in a drip-and-ship model compared to that for patients treated with the mothership paradigm (293 vs. $232 \mathrm{~min}, \mathrm{p}=0.03$ ). The 30 -day mortality rates and functional outcomes were similar in the COVID-19 and pre-COVID-19 periods.

\section{Discussion}

We conducted an exploratory analysis to estimate the change in the number of new stroke diagnoses in our region, and to evaluate the impact of the COVID-19 pandemic on major stroke quality measures.

Our study showed that the stroke care system in the Masovian Voivodeship was significantly affected by COVID-19, with a $20 \%$ decrease in acute cerebrovascular accident admissions. The World Stroke Organisation reported a global reduction of $42 \%$ in acute cerebrovascular accident admissions [4]. A recent paper revealed a decrease in general stroke admissions of $19.4 \%$ and a reduction in stroke unit admissions of $24.6 \%$ from January to May 2020 compared to the same months in 2019 in Malopolska Voivodeship. Moreover, the number of patients treated with interventional services declined during the COVID-19 pandemic [-32\% for intravenous recombinant tissue plasminogen activator (rtPA) and -25\% for mechanical thrombectomy] compared to the previous year. The exact figures, including stroke quality measures, were not provided [5].

Decreases in the number of acute stroke admissions could be caused by fear of COVID-19. Strict social distancing rules persuaded patients to avoid face-to-face medical consultation. Additionally, isolation could also have an impact through reducing the chance of another individual noticing stroke symptoms [2]. The decrease in the number of admissions to stroke departments during the COVID-19 pandemic observed in our study was mainly related to fewer admissions due to TIAs. TIA patients have a high short-term risk of developing ischaemic stroke, especially within the first 48 hours, so a public education campaign raising awareness of stroke as well as TIA signs, the need for timely diagnosis, and urgent preventive treatment, are all essential to reduce this risk.

The major problems revealed in our study involved pre- and in-hospital delays. Factors causing delays were the COVID-19 screening process and the reallocation of medical staff and protective equipment to COVID-19 care, which led to a shortage of resources at non-COVID hospitals and an inability to create or maintain fast-track stroke-care channels [2]. Moreover, healthcare professionals faced physical and mental pressure due to the continuously high risk of SARS$\mathrm{CoV}-2$ infection and were overworked because of quarantined or relocated colleagues $[6,7]$.

COVID-19 testing should not cause any impact on or delay to stroke diagnostics or treatment. Therefore, some authors have suggested the addition of chest CT to standard stroke protocols, which could identify pulmonary COVID-19 complications, and/or the implementation of fast screening, for example rapid antigen testing for SARS-CoV-2, the results of which may be later confirmed with RT-PCR testing. Furthermore, every patient must be treated as potentially infected and wear a mask throughout the whole diagnostic pathway, with the exception of a facial nerve palsy examination to facilitate appropriate NIHSS evaluation [2]. The introduction of masks and antigen testing in our stroke centres improved the in-hospital flow of patients and boosted the sense of safety among staff. However, exact data on how these measures affected quality metrics in stroke care is lacking. 
Table 1. Clinical characteristics of studied cohort

\begin{tabular}{|c|c|c|c|}
\hline & Pre-COVID-19 & COVID-19 & $\mathbf{P}$ \\
\hline$n(\%)$ & $233(55.6)$ & $186(44.4)$ & \\
\hline $\operatorname{Sex}(F) n(\%)^{*}$ & $124(53.2)$ & $88(47.3)$ & 0.2 \\
\hline Age mean $( \pm S D)^{*}$ & $71.9(13.6)$ & $69.8(13)$ & 0.1 \\
\hline Acute cerebrovascular incident admissions $/ 100,000$ inhabitants in study period & 29.125 & 23.25 & 0.04 \\
\hline \multicolumn{4}{|l|}{ Reason for acute cerebrovascular hospital admission } \\
\hline TIA & $45(19.3)$ & $20(10.7)$ & 0.01 \\
\hline non-TIA & $188(80.7)$ & $166(89.2)$ & - \\
\hline Ischaemic stroke & $170(72.9)$ & $153(82.2)$ & 0.2 \\
\hline Intracerebral haemorrhage & $18(7.7)$ & $11(5.9)$ & 0.2 \\
\hline $\mathrm{SAH}$ & 0 & $2(1)$ & - \\
\hline \multicolumn{4}{|l|}{ Ischaemic stroke aetiology } \\
\hline LAD & $50(23.2)$ & $46(26.13)$ & 0.2 \\
\hline CS & $68(31.6)$ & $42(23.8)$ & - \\
\hline SVD & $48(22.3)$ & $47(26.7)$ & - \\
\hline LVO** & $77(33)$ & $56(30)$ & 0.7 \\
\hline Intravenous rtPA & $68(29.2)$ & $54(29)$ & 0.9 \\
\hline DTN & $29.7(21)$ & $48(23.5)$ & $<0.01$ \\
\hline DTCT & $25.9(10)$ & $37.7(25)$ & $<0.01$ \\
\hline rtPA+ mechanical thrombectomy & $27(11.6)$ & $30(16)$ & 0.1 \\
\hline Mechanical thrombectomy & $39(16.7)$ & $34(18.3)$ & 0.6 \\
\hline NIHSS $>8 p$ & $77(45)$ & $71(47)$ & 0.9 \\
\hline NIHSS admission & $11.9(8)$ & $10.2(7)$ & 0.05 \\
\hline NIHSS discharge & $6.3(7)$ & $4.9(5)$ & 0.06 \\
\hline $\mathrm{mRS}$ discharge & $3.3(2)$ & $3.1(2)$ & 0.2 \\
\hline mRS admission & $4.2(3)$ & $4.4(2)$ & 0.6 \\
\hline mRS 30 day & $4.3(2)$ & $4.6(2)$ & 0.4 \\
\hline 30-day mortality & $35(15)$ & $21(11.5)$ & 0.7 \\
\hline DTCT & $27.1(10)$ & $41.2(27)$ & 0.04 \\
\hline DTG (drip-and-ship) & $35(41)$ & $73(76)$ & 0.04 \\
\hline DTG (mothership) & $75.9(56)$ & $123.7(75)$ & 0.03 \\
\hline OTG (all patients) & $223(71)$ & $259(80)$ & $<0.01$ \\
\hline OTG (drip-and-ship) & $240(69)$ & $293(51)$ & 0.01 \\
\hline OTG (mothership) & $203(74)$ & $232(104)$ & 0.04 \\
\hline Effective recanalisation (2b, 3) n (\%) & $27(73)$ & $24(88.8)$ & 0.11 \\
\hline 30-day mortality n (\%) & $9(24)$ & $9(28)$ & 0.7 \\
\hline 30-day mRS mean ( $\pm \mathrm{SD})$ & $3.8(2.1)$ & $2.9(2.4)$ & 0.14 \\
\hline NIHSS admission & $17(6)$ & $15.5(5)$ & 0.1 \\
\hline NIHSS discharge mean ( \pm SD) & $11(10)$ & $6.3(4.1)$ & 0.02 \\
\hline
\end{tabular}

${ }^{*}$ Data expressed as means ( \pm SD) or numbers (\%); * $V$ S. no LVO; TIA - transient ischaemic attack; SAH - subarachnoid haemorrhage; LAD - large artery atherothrombotic disease; CS - cryptogenic stroke; SVD - cerebral small vessel disease; LVO — large vessel occlusion; rtPA - recombinant tissue plasminogen activator; DTN - door-to-needle; DTCT — door-to-computed tomography; NIHSS - National Institutes of Health Stroke Scale; mRS — modified Rankin scale; DTG — door-to-groin; OTG — onset-to-groin

Mechanical thrombectomy is provided in Poland via two main organisational paradigms: the mothership, in which the patient is directly brought to a CSC, and the drip-and-ship model, in which initial assessment and eventual thrombolytic treatment at the primary stroke centres are followed by 'shipping' out to a CSC. The choice of one model over the other implies clinical consequences for treated patients as well as for local health policies, including the distribution of hospital facilities over the region of interest.

Our data shows that the mothership model provided significantly shorter OTG delays, of more than one hour. This may indicate that it might be considered the model of choice during the pandemic. Avoidance of additional triage in overcrowded emergency 


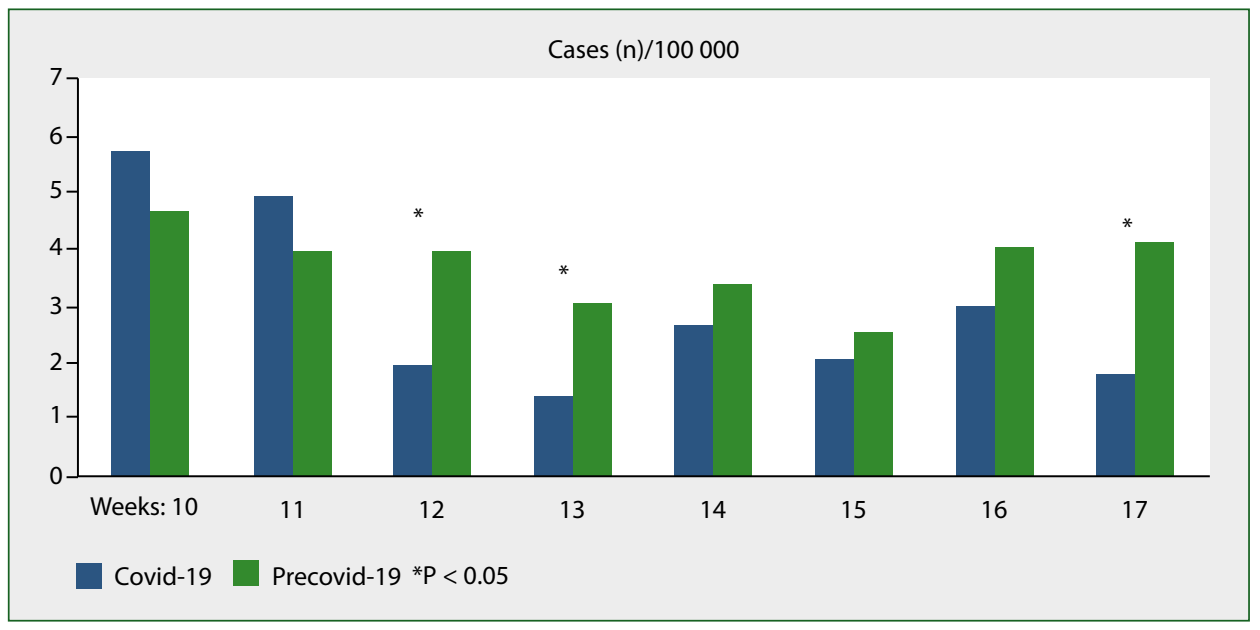

Figure 1. Acute cerebrovascular incident admissions during COVID-19 pandemic compared to those during same period in 2019

departments, and arranging transport, both of which are necessary in the drip-and-ship model, could prevent delays caused by a lack of resources that are otherwise engaged in COVID-19 care.

In November and December 2020, Poland's healthcare system struggled with the second wave of the coronavirus pandemic, with the number of new daily cases reaching over 27,000 , and a national quarantine was expected. The limitation of the presented study is that the results might not be representative of other regions of Poland. Although we did not find a significant difference in stroke outcomes between patients treated during the COVID-19 and pre-COVID-19 periods, this might have been related to limited sample size. The reason for the difference in the number of stroke admissions between the weeks of the COVID-19 pandemic and the frequency of recent TIA in patients hospitalised due to stroke have not been analysed in our study, so a follow-up study looking at these metrics in the second wave would be important.

However, due to the limited number of other studies, our data might be important in terms of optimising acute stroke care before a third wave and until a COVID-19 vaccine becomes widely available.

\section{Future directions}

Our study shows that even countries less affected by the COVID-19 pandemic during the first wave, such as Poland, experienced collateral adverse effects on stroke-care quality metrics. The onset of the COVID-19 pandemic was correlated with a reduction in the number of admissions to stroke departments, and resulted in a significant increase in stroke interventional treatment delays. To combat this trend, optimising healthcare resources before subsequent pandemic waves is necessary. These actions should include reorganisation of the stroke-care network, with promotion of the mothership paradigm; the development of new in-hospital care pathways based on continuous analysis of local data; and nationwide education campaigns about the importance of immediate response to stroke and TIA symptoms. Moreover, a campaign is required to reassure patients that hospitals provide safe in-person medical care in cases of alarming symptoms.

As these findings are preliminary conclusions from our data, the full impact of the COVID-19 pandemic on stroke management in Poland still needs to be evaluated. Furthermore, studies evaluating the impact of peri-stroke COVID-19 on the risk and aetiology of stroke are needed.

Ethical permissions: Ethical approval was not necessary for the preparation of this article.

Funding: This publication was prepared without any external funding source.

\section{References}

1. Markus HS, Brainin M. COVID-19 and stroke-A global World Stroke Organization perspective. Int J Stroke. 2020; 15(4): 361-364, doi: 10.1177/1747493020923472, indexed in Pubmed: 32310017.

2. Zhao J, Li H, Kung D, et al. Impact of the COVID-19 Epidemic on Stroke Care and Potential Solutions. Stroke. 2020; 51(7): 1996-2001, doi: 10.1161/STROKEAHA.120.030225, indexed in Pubmed: 32432997.

3. Szmuda T, Ali S, Słoniewski P, et al. Telemedicine in neurosurgery during the novel coronavirus (COVID-19) pandemic. Neurol Neurochir Pol. 2020; 54(2): 207-208, doi: 10.5603/PJNNS.a2020.0038, indexed in Pubmed: 32319670.

4. Liu R, Zhao J, Fisher M. The global impact of COVID-19 on acute stroke care. CNS Neurosci Ther. 2020 [Epub ahead of print], doi: 10.1111/ cns.13442, indexed in Pubmed: 32725844.

5. Słowik A, Nowak R, Popiela T. Significant fall in stroke admissions in the Malopolska Voivodeship of Poland during the COVID-19 pandemic. Neurol Neurochir Pol. 2020; 54(5): 471-472, doi: 10.5603/PJNNS. a2020.0056, indexed in Pubmed: 32700757.

6. Remuzzi A, Remuzzi G. COVID-19 and Italy: what next? The Lancet. 2020; 395(10231): 1225-1228, doi: 10.1016/s0140-6736(20)30627-9.

7. Zaka A, Shamloo SE, Fiorente P, et al. COVID-19 pandemic as a watershed moment: A call for systematic psychological health care for frontline medical staff. J Health Psychol. 2020; 25(7): 883-887, doi: 10.1177/1359105320925148, indexed in Pubmed: 32370621. 